\title{
LA CUEVA DEL MIRÓN (RAMALES DE LA VICTORIA, CANTABRIA): EXCAVACIONES 1996-1999
}

\author{
THE MIRÓN CAVE (RAMALES DE LA VICTORIA, CANTABRIA): EXCAVATIONS 1996-1999
}

\author{
MANUEL R. GONZÁLEZ MORALES $(*)$ \\ LAWRENCE G. STRAUS $(* *)$
}

\section{RESUMEN}

Las cuatro campañas de excavación desarrolladas en la Cueva del Mirón, con una estratigrafía continua que abarca por ahora desde el Bronce inicial hasta el Solutrense, han puesto de manifiesto la importancia de este yacimiento para el conocimiento de las sociedades humanas de las áreas interiores de la Región Cantábrica a lo largo de buena parte de la Prehistoria. Los procedimientos de excavación aplicados han permitido documentar en detalle espacios domésticos del Calcolítico, el Neolítico y el Paleolítico Superior final y recoger gran cantidad de testimonios industriales y ambientales, junto con muestras de arte parietal paleolítico. Las cuarenta dataciones radiocarbónicas obtenidas hasta el momento la convierten en la secuencia más completa del Cantábrico, incluyendo una interesante serie de fechas para los momentos iniciales del Neolítico regional. Los datos de la prospección geofísica señalan una potencia sedimentaria que asegura la posible continuación en profundidad de los depósitos arqueológicos.

\footnotetext{
ABSTRACT

The four excavation campaigns carried out to date in $\mathrm{El}$ Miron Cave, with a complete stratigraphic sequence from the early Bronze Age through theSolutrean, have demonstrated the importance of this site to the understanding of human societies in the montane interior of the Cantabrian Region during much of its prehistory. The excavation methods that have been applied especially document in detail residential areas pertaining to the Chalcolithic, Neolithic, and early-mid Magdalenian periods within this large cave. They also have provided large quantities of paleoenviron-

(*) Dpto. de Ciencias Históricas. Universidad de Cantabria. 39071 Santander. España.

(**) Dept. of Anthropology. University of New Mexico. Albuquerque, NM 87131 USA.

El artículo fue remitido en su versión final el 3-IV-2000.
}

mental and cultural data that are currently under analysis. Numerous examples of parietal engravings have been discovered and, in some cases, stratigraphically dated to the Magdalenian. The forty radiocarbon dates obtained so far already constitute the largest series for any Cantabrian site and include a number of important dates for the beginning of the regional Neolithic sequence at c. 5800 BP (uncal.). Results of geophysical survey indicate the existence of a sedimentary infilling in the cave vestibule that could include archeological deposits well below the 19,000 year-old Solutrean layers reached so far.

Palabras clave: Calcolítico. Neolítico.Aziliense. Magdaleniense. Solutrense. Radiocarbono. Región Cantábrica. Arte parietal paleolítico.

Key words: Chalcolithic. Neolithic. Azilian. Magdalenian. Solutrean. Radiocarbon. Cantabrian Region. Palcolithic rock art.

\section{INTRODUCCIÓN}

La Cueva del Mirón, situada en Ramales de la Victoria (Cantabria) (Fig. 1), vecina de yacimientos de arte paleolítico tan conocidos como las cuevas de Covalanas, situadas escasamente una treintena de metros sobre ella, La Haza o Cullalvera, todas ellas situados en el Monte Pando, aparece mencionada en los primeros inventarios de yacimientos de la zona cantábrica a principios del siglo XX (Alcalde del Río, 1906; Alcalde del Río et alii, 1911; Cabré, 1915; Obermaier, 1916, 1924; Sierra, 1908) con citas al aparente volumen y riqueza de su yacimiento paleolítico, aunque también algunos autores hablan de la remoción que mostraba. Quizá por este último aspecto, ya en la segunda edición de El Hombre Fósil de Obermaier se omite en los 


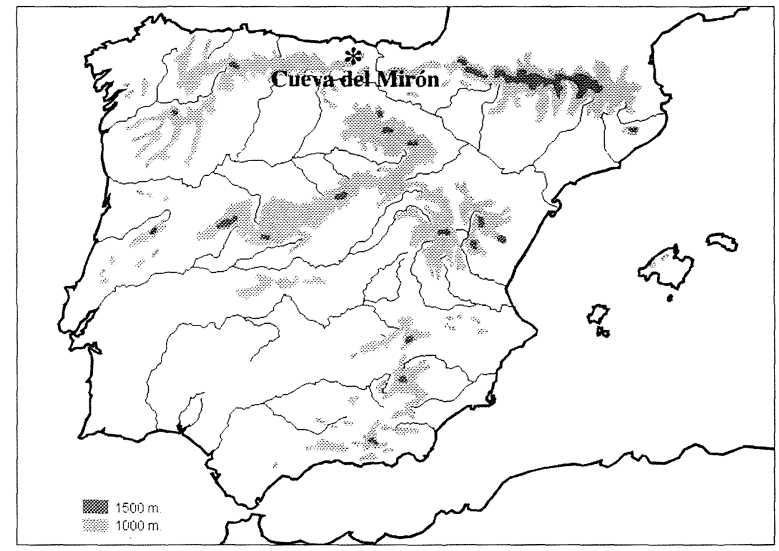

Fig. 1. Localización de la Cueva del Mirón (Ramales de la Victoria, Cantabria).

listados y cae prácticamente en el olvido durante más de setenta años. En otro lugar (González Morales y Straus, 1997) hemos hecho referencia a estas cuestiones historiográficas con más detalle, así como a la relevancia de la situación de la Cueva del Mirón como posible yacimiento clave para entender el poblamiento prehistórico de la zona oriental de Cantabria y las relaciones costa/interior montañoso a una escala más general.

En ese mismo lugar se recogen las principales hipótesis generales de partida de nuestro proyecto de investigación, centradas en la comprensión de las estrategias de uso de recursos, producción, movilidad y organización social de los grupos humanos de la Prehistoria en la zona, así como de la relación con los yacimientos con arte parietal de Ramales y la definición del proceso de cambio al Neolítico en un área en la que contamos con el testimonio de monumentos megalíticos inmediatos a la zona de la cueva. La primera campaña, como más adelante se señala, dedicada a explorar las posibilidades del sitio, nos mostró la potencialidad de la larga secuencia de la Cueva del Mirón para contribuir a dar respuesta a las cuestiones planteadas y llevarnos a la formulación de otras nuevas.

Nuestra intención es presentar aquí un breve resumen de lo realizado en las cuatro campañas de excavación llevadas a cabo en el yacimiento entre 1996 y 1999; campañas que han tenido una duración de 45 días en el primer año y de 60 días en los restantes y han sido desarrolladas por un equipo de trabajo que en las últimas campañas integra por término medio a unas 22 personas de manera regular entre la cueva y el laboratorio de campo instalado en Ramales. Se han venido publicando resúme- nes preliminares de los resultados de cada campaña (González Morales y Straus, 1997; Straus y González Morales, 1996, 1997, 1998a, 1999), así como algunas revisiones más generales (Straus y González Morales, 1998b, 1998-99), pero ahora pretendemos dar a conocer los datos básicos del conjunto de las cuatro campañas, que cierran una etapa de trabajo en este singular yacimiento.

\section{SITUACIÓN DEL YACIMIENTO}

La Cueva del Mirón se ubica en un acantilado calizo en la falda Oeste del monte denominado El Pando, contrafuerte de la Peña del Moro que domina la confluencia de los ríos Calera y Gándara y la vega al final de la que éste entrega sus aguas al Asón, donde se sitúa la localidad de Ramales de la Victoria. La posición de la cueva es estratégica, pues domina el paso de las grandes vías de comunicación antiguas y actuales entre la costa y el valle principal del Asón con la Meseta y con el extenso valle de Soba. El acceso a Castilla a través del Puerto de los Tornos, a $920 \mathrm{~m}$ de altitud, ha sido una ruta tradicional de movimiento desde la costa al interior a lo largo de toda la Historia, y el valle del Río Carranza, que se une alAsón aguas abajo de Ramales, es a su vez la gran vía de comunicación hacia Las Encartaciones y el País Vasco, por donde todavía hoy circula el ferrocarril a Bilbao. Esta confluencia de valles de montaña es, por tanto, una verdadera encrucijada en el interior de la Región Cantábrica, ligando Cantabria central y oriental con Vizcaya, por una parte, y la costa con el Valle del Ebro y la Meseta por otra (Fig. 1).

La amplia boca de la Cueva del Mirón se abre a unos $250 \mathrm{~m}$ sobre el nivel del mar actual y $150 \mathrm{~m}$ sobre el fondo del valle, mirando al Oeste y frente al Pico San Vicente, un punto de referencia extraordinario para posibles agregaciones humanas, que es visible junto con la Peña del Moro tanto desde la costa como desde la divisoria cantábrica. El vestíbulo de la cueva (Fig. 2) mide $18 \mathrm{~m}$ de ancho por 30 $\mathrm{m}$ de fondo y unos $12 \mathrm{~m}$ de altura en su punto máximo. En el fondo del vestíbulo se encuentra la base de un empinado depósito de arcilla y cantos que se eleva hacia la galería interior de la cueva, un pasadizo de un centenar de metros, relativamente recto y de unos $8 \mathrm{~m}$ de ancho y unos $3 \mathrm{~m}$ de altura máxima. El techo de la cueva, prácticamente horizontal en todo su desarrollo, muestra los llamados "golpes de gubia", testigo de su formación inicial bajo con-

T. P., 57, n. ${ }^{\circ} 1,2000$ 


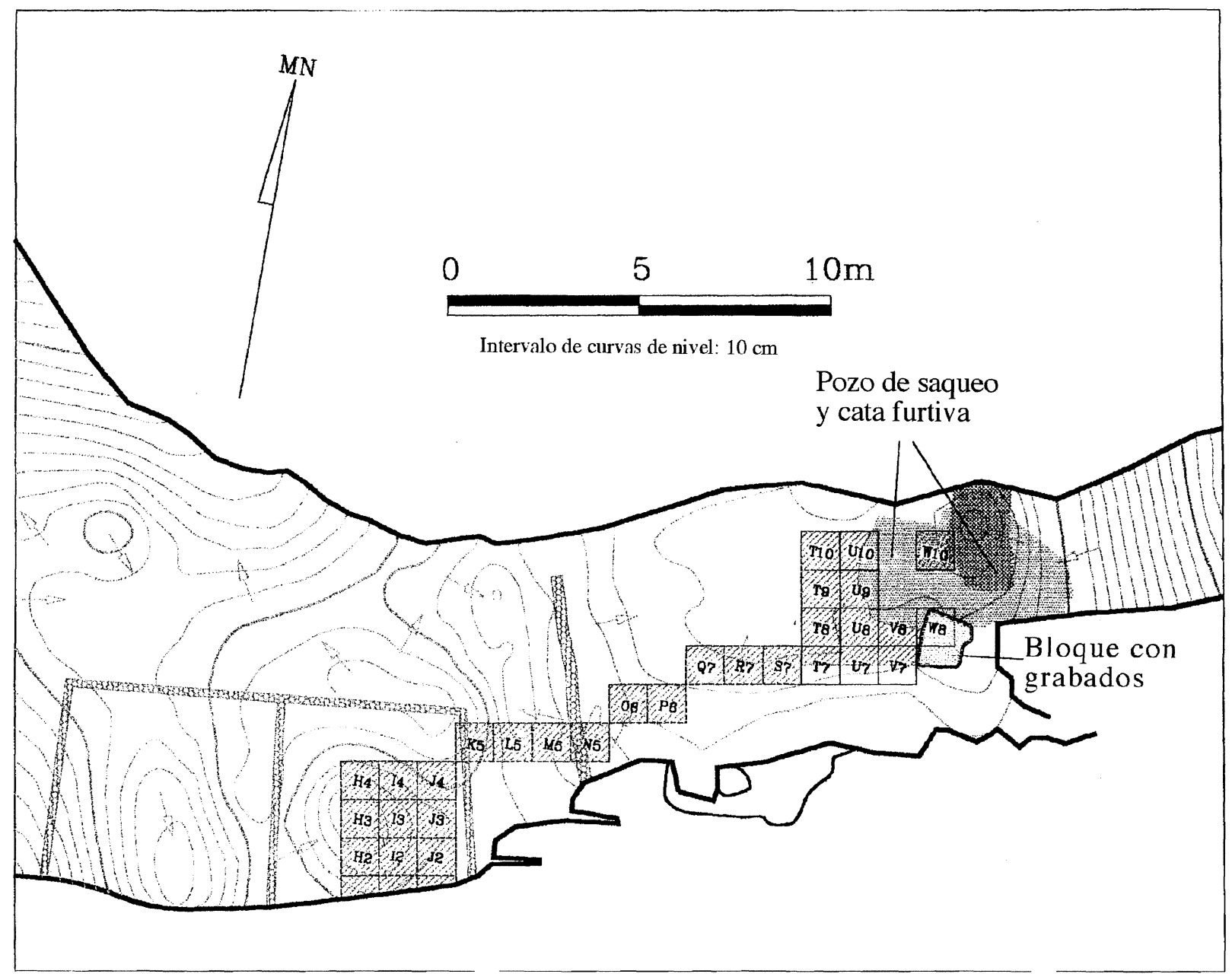

Fig. 2. Cueva del Mirón. Ramales de la Victoria (Cantabria, España). Topografía: Eduardo Torres.

diciones de presión, y a la vez de su gran estabilidad, casi sin desprendimientos identificables. A juzgar por el contorno y la pendiente de las huellas de erosión posteriores de las paredes de la cueva, a caudal libre, tanto el vestíbulo como la galería parecían contar con rellenos sedimentarios muy profundos. El frente del vestíbulo, al exterior o bajo la zona inicial de la visera, está sembrado de grandes bloques de derrumbe de la bóveda, que toma en ese punto el característico perfil de equilibrio, pero su parte central y fondo tienen un techo plano del cual cuelgan algunas estalactitas de gran tamaño y muy alteradas, indicio de la falta de caída de bloques en un período prolongado. Por estos motivos, la Cueva del Mirón parecía ofrecer unas condiciones excepcionales para la preservación de un gran yacimiento adecuado para cubrir los objetivos previstos en nuestro proyecto, lo que nos movió a iniciar su excavación.

\section{DESCRIPCIÓN DE LOS TRABAJOS}

Al inicio de la excavación, el vestíbulo de la cueva era aún utilizado como aprisco para cabras, y los informante locales nos indicaron que había tenido en el pasado reciente un uso intensivo para estabular ganado ovino y caprino. Con anterioridad -posiblemente en el siglo XIX-se habían construido en la entrada de la cueva dos grandes cabañas adosadas a la pared y unidas por uno de sus lados, de las que se conservaba tan sólo la base de las paredes. Los cascotes producto de su derrumbe habían sellado los depósitos sobre las que se habían edificado, asegurando su protección al menos contra furtivos arqueológicos actuales. En cambio, en el resto de la entrada de la cueva, las prácticas de extracción de abono determinaron la remoción de la capa superficial en la mayor parte de su extensión. Este hecho, unido a la nivelación del suelo para la 
instalación de corrales de ganado, han provocado la desaparición de los hipotéticos niveles de la Prehistoria reciente en la zona del fondo del vestíbulo, como veremos. En esta misma zona aún se veían los restos de una cata de furtivos de unos $2 \mathrm{~m}^{2}$, ya casi rellena de sedimentos, que había sido realizada a principios de los años 80. En la galería interior de la cueva, a unos $60 \mathrm{~m}$ de la entrada, existía una trinchera transversal de aproximadamente $5 \mathrm{~m}$ de largo por metro y medio de ancho y un metro de profundidad, excavada a finales de los años 50 por los obreros que realizaron la carretera de acceso a la cueva de Covalanas, por orden de los entonces responsables del tema en la Diputación Regional, para verificar la posible existencia de yacimiento (1). En la parte superior del corte de esa trinchera, muy deteriorado por rebuscas, se veía un nivel carbonoso fino que parecía extenderse a todo lo largo de la sección y continuarse en otra cata cercana.

A partir de esta situación, las excavaciones de sondeo de 1996 se centraron en tres zonas: bajo una de las antiguas cabañas derrumbadas de la entrada de la cueva ("zona de la cabaña"), en el antiguo corral de cabras del fondo del vestíbulo ("zona del corral") y en la galería interior de la cueva. En todas las zonas se localizaron depósitos de ocupación humana. La práctica totalidad del sedimento se ha cribado en agua, en 1996 en la propia cueva y en las campañas siguientes se ha bajado toda la tierra al laboratorio de campo, en Ramales, para someterla a flotación y lavado para su posterior selección a mano. En la cueva se han coordenado individualmente todos los útiles reconocidos in situ, los restos de talla de más de $1 \mathrm{~cm}$ y fragmentos de hueso superiores a $5 \mathrm{~cm}$ y todos los fragmentos óseos identificables percibidos al excavar, así como todos los instrumentos óseos y una parte significativa de la cerámica. Desde 1997 se ha venido aplicando un procedimiento experimental de registro mediante fotogrametría digital, desarrollado porA. Astorqui, que permite la generación de planos completos de las áreas de excavación combinados con la recogida sobre foto digital impresa en la cueva, que supone un importante ahorro de tiempo y trabajo rutinario. Y desde la campaña de 1999 se ha unificado toda la toma de coordenadas en la excavación mediante estación total, lo que ha proporcionado una mayor precisión en el registro.

En la zona de la cabaña se excavó una superficie inicial de $4 \mathrm{~m}^{2}$, reducidos posteriormente a tres

(1) Referencias facilitadas por Jesús Gutiérrez, anterior guía de la cueva de Covalanas. por necesidades de tiempo, pero que fue suficiente para determinar la existencia de un paquete superior de niveles integrados por numerosos hogares con cubetas excavadas en el suelo y relleno de fragmentos de roca, abundante carbón y cenizas, y restos de fauna, y alrededor de los cuales también aparecían huesos de animales (especialmente ovicaprinos, bóvidos y cerdos), instrumental lítico y fragmentos de cerámica, tanto de vasos de pastas relativamente cuidadas, superficies espatuladas y paredes finas, como de grandes vasijas de almacenamiento de factura mucho más grosera, toda ella sin decorar. Este paquete estratigráfico (sobre todo los niveles 4 a 7 y las estructuras incluidas en los mismos) incluía puntas de retoque plano, una de ellas de aletas y pedúnculo y otra de base convexa, que junto con la cerámica apuntaban a una cronología calcolítica más tarde confirmada por las dataciones absolutas. Por debajo se desarrollaba una serie de estratos de matriz más arcillosa, alternando o incluyendo lentejones carbonosos de hogares someros, realizados en su mayoría sobre el suelo, sin excavar cubetas, en los que también abundaban los restos de fauna, ahora con aparente dominio de ovicaprinos y ciervo. Las cerámicas eran en general más finas, correspondientes a vasos más pequeños y por lo general de pastas cuidadas, también sin decorar salvo el caso de un pequeño mamelón de un fragmento del nivel 8.1. En la industria lítica faltaban por completo los elementos de retoque plano, y los útiles retocados eran escasos y poco diagnósticos. Esta serie de ocupaciones correspondía al Neolítico, abarcando casi un milenio de habitación en la cueva, como ahora sabemos.

Por debajo de los niveles con cerámica se prosiguió excavando un primer estrato que continuaba casi sin distinción la base del último nivel neolítico (el nivel 10) rico en depósitos carbonatados, pasando luego a un estrato de matriz más arcillosa en cuya base aparecía un amplio lentejón carbonoso (niveles 11 y 11.1) con abundantes puntas de dorso y disquitos raspadores de tipo aziliense, seguido de otro estrato progresivamente más arcilloso (nivel 12) en el que se recogió un fragmento de arpón de una hilera de dientes. La secuencia continuaba con dos potentes estratos de arcillas plásticas (niveles 13 y 14), relativamente pobres en restos de fauna e industria, para llegar a la base del sondeo excavado en esta campaña, el nivel 15, donde aparecía un auténtico suelo de ocupación con plaquetas calizas y abundantes testimonios líticos y faunísticos, correspondiente al Magdaleniense inferior. 
A partir de 1997, tras terminar de excavar el cuadro I3 dentro del sondeo del año anterior, se extendió el área de trabajo hacia el oeste del sondeo (cuadros H1-4) y hacia el norte, en dirección al eje de la cueva (cuadros H4/I4/J4), dejando expuesta un área de $10 \mathrm{~m}^{2}$. En esta campaña y sucesivas se han ido excavando estos nuevos cuadros (niveles 2 a 7 en 1997,8 a 10.1 en 1998 y 11 a 12 en 1999), a la vez que se continúa profundizando en el fondo del sondeo, donde se han excavado los niveles 15, 16 y 17. Es necesario señalar que, salvo las alteraciones producidas por las estructuras antrópicas (cubetas del conjunto superior de niveles del Bronce inicial 3 a 3.5, hogares calcolíticos de los niveles 4 a 7), el depósito es muy regular, sin grandes bloques desprendidos o huellas de erosión evidentes, lo cual ha determinado la excepcional buena conservación de materiales y estructuras.

En la zona del corral, al fondo del vestíbulo, la situación era más compleja. Al vaciar la cata de furtivos ya mencionada, se pudo apreciar que la zona revuelta no se reducía a sus límites aparentes, sino que había sido realizada dentro de una zona de mayor extensión ya removida en profundidad en el pasado, aunque sellada por capas compactadas de tierra y excrementos de ganado ovino, lo que le otorgaba una cierta antigüedad. En esa campaña y sucesivas se pudo definir que se trataba de un gran pozo de planta irregular, de unos $4 \mathrm{~m}$ de diámetro aproximado, donde se había excavado hasta metro y medio de profundidad en algunas zonas, llegando a picar y romper bloques calizos incluidos en el sedimento. Esta actuación sistemática y costosa en términos de tiempo y esfuerzo solamente puede ser debida a buscadores de tesoros, que no es extraño que actúen en este tipo de cuevas visibles y de grandes dimensiones. Junto a ello, la mayor parte de la superficie de esa zona de la cueva fuera del pozo estaba removida hasta aproximadamente medio metro de profundidad, creando una capa de cascajo sin apenas matriz terrosa, pero que incluía abundantes restos de industria lítica y ósea prehistóricas, junto con cerámicas y otros materiales de origen reciente. Esta capa ha sido producida por la práctica, habitual en cuevas donde ha habido estabulación de ganado, de extraer la tierra orgánica como abono, cribándola in situ y dejando allí el resto, desarrollada en El Mirón hasta fecha reciente según los informantes locales. Por fortuna, como más tarde comprobamos, esta acción había afectado solamente a la zona superficial ya mencionada.
En la zona de excavación abierta en 1996 en la zona del corral (cuadros T-U-V/7-8 inicialmente, luego reducidos a V8 y la mitad norte de V7), tras limpiar parcialmente el revuelto, se localizaron desde superficie intacta niveles atribuidos al final de Paleolítico Superior, donde la riqueza de materiales era muy superior a la de la zona de la cabaña. En los estratos más recientes de la serie intacta -niveles 102 a 104- se recuperaron útiles y numerosos restos de talla, a pesar de la reducida superficie excavada, cuyo número se multiplicaba en los niveles subyacentes 105 a 108. En éstos también eran extremadamente abundantes los restos de fauna, normalmente ennegrecidos en el nivel 108, de matriz orgánica muy oscura.

Las campañas siguientes permitieron confirmar el interés de esta zona de la excavación, ampliándola en 1997 a la superficie inicialmente abierta hasta alcanzar el nivel 108, en tanto que en el cuadro V8 se continuaba excavando como sondeo la serie de niveles 108-116; en 1998 se siguió excavando una secuencia extraordinariamente rica de niveles magdalenienses (niveles 108 a 112) en los cuadros UV7 y U8 que suministraron grandes cantidades de restos de talla lítica (sobre todo lasquitas de retoque y pequeñas hojitas) y útiles, incluyendo geométricos. Las materias primas líticas incluyen una amplia variedad de sílex y calcedonia, en general de excelente calidad. Los restos de fauna eran también muy abundantes, incluyendo cráneos y cornamentas de cabra y abundantes testimonios de esta especie y de ciervo, si bien en delicado estado de conservación en ocasiones. Cerca del límite de los cuadros T-U/7-8 se localizó, sobre el nivel 112, un aparente "murete" rectilíneo de piedras que se dejó in situ con la intención de abrir la zona inmediata para poder comprender mejor el conjunto de esta posible estructura. Como cierre de la campaña en esta zona se realizó un sondeo en el cuadro W10, en el fondo del pozo de buscadores de tesoros, para identificar los niveles subyacentes (provisionalmente numerados de 400 en adelante, y de manera definitiva designados 120 a 128). En este sondeo se identificaron una serie de estratos con numerosos restos de plaquetas de gelifracción, intercalados con superficies de ocupaciones en las que se recogieron diversos tipos de puntas solutrenses y numerosos colgantes, junto a algunos útiles de asta y escasos restos de fauna. Por último, en 1999 se ha excavado la parte conservada de los cuadros T/8-10 y U/ 9-10, al borde del pozo de saqueo, a la vez que se continuaba el sondeo en V8 hasta alcanzar el fon- 
do de este pozo y enlazar la base de la estratigrafía de este corte con el arranque del sondeo deW10. En los niveles 118 y 119 se recogieron contadas piezas con retoque invasor, pero cabe señalar que la existencia de varias cubetas excavadas en ellos nos obliga a tener precaución a la hora de atribuir esos materiales con seguridad.

En esta misma zona del corral se localizó un gran bloque desprendido con grabados lineales de trazo profundo, situado en estratigrafía y con la base de los grabados recubierta por el nivel 104, lo cual certificaba el límite ante quem de su realización, en tanto que el bloque reposaba sobre el nivel 110, hito post quem a su vez, dado que los grabados solamente pudieron ser ejecutados una vez caído el bloque. Su contexto más probable, por tanto, se sitúa entre fines del Magdaleniense inferior y un momento avanzado del Magdaleniense medio, en términos de unidades cronoestratigráficas convencionales. Cerca de él, en la pared sur, se identificaron más líneas grabadas de trazo profundo, en parte recubiertas por el sedimento, así como otros grabados más finos también recubiertos parcialmente en la pared oriental. En la pared norte de esta misma zona, una limpieza de la suciedad que recubría una reducida zona en 1999 ha puesto al descubierto grabados de trazo muy fino que incluyen al menos dos figuras de animales. Dada la amplia extensión de pared aún cubierta de suciedad, cabe esperar que estos grabados se continúen por la zona.

En 1998 se abrió un nuevo sondeo en el centro del vestíbulo de la cueva (cuadros O-P/6), con la intención de comprender la relación de las secuencias estratigráficas de ambas zonas principales de excavación, separadas por $9 \mathrm{~m}$ de distancia, que se continuó en ambas direcciones en 1999 (desplazándolo un metro en cada sentido para poder realizar la unión física de la secuencia, ver Fig. 2). El cuadro K5 solamente se excavó en su parte superior para evitar problemas de estabilidad a los cortes de la zona de la cabaña. De los cuadros L5 a P6, bajo un depósito superficial de relleno revuelto y mezclado (nivel 300) y un segundo nivel parcialmente revuelto, pero más limoso (301), hay una capa más compacta de limos finos arenosos con algunas gravas y cantos, ya sin materiales modernos (nivel 302). La serie 303.1 a 303.3. incluye diversos lentejones diferenciados como restos de hogueras sobre una capa inferior de travertino (nivel 304), que es aparentemente estéril. Éste a su vez recubre un depósito granular de limos arenosos, blanquecino y enriquecido con carbonatos de calcio precipitados (nivel 305). También es virtualmente estéril desde el punto de vista arqueológico. El nivel más bajo alcanzado en este sondeo en 1998 ha sido el 306, un limo arenoso granular de gris a pardo oscuro, con piezas de sílex, huesos, cantos y éboulis angulosos. En parte de la zona excavada hay diversos pozos y cubetas que cortan la estratigrafía, algunos antiguos y sellados por niveles prehistóricos y otros que arrancan de la superficie actual y corresponden a remociones más recientes.

En dirección Este, se abrieron los cuadros Q-R$\mathrm{S} / 7$, donde bajo los niveles revueltos superficiales se alcanzaba la continuación del nivel 101 del corral como primer estrato intacto, y bajo él aparecía la superficie de 102, punto en el que se interrumpió el sondeo por estimar cubiertos los objetivos previstos. A falta de la conexión total (sólo posible si se excavara el cuadro K5 en su totalidad), nuestra hipótesis a partir de la observación de la estratigrafía, los materiales recuperados y las dataciones de radiocárbono es que, de arriba abajo, 301.1 y 301.2 corresponden a las ocupaciones calcolíticas de la zona de la cabaña (presumiblemente se correlacionan con los niveles 6 y 7), en tanto que la serie de 303 a 303.3 encaja con los niveles neolíticos. Por su composición, el nivel 304 parece haberse formado bajo las condiciones húmedas delAtlántico y podría ser equivalente a lo que resta del nivel 101 de la zona del corral, en tanto que el subyacente nivel 305 posiblemente se relaciona con el nivel 102 de esta última zona, y ambos se podrían a su vez ligar a la serie de $11,10.1$ y base del 10 de la zona de la cabaña. El nivel 306 enlaza aparentemente con 102.1 del corral y con mucha probabilidad se corresponde con 11.1 de la cabaña, datados todos en el intervalo 11950/11650 BP.

La última zona de trabajo abordada en el inicio de las excavaciones del Mirón fue la ya mencionada trinchera excavada a fines de los años cincuenta en la galería interior de la cueva. Tras establecer la cuadrícula de referencia (cuadros 8-12/Q-R-S) se fue recogiendo el sedimento revuelto del interior de la trinchera, procedente sobre todo de las remociones efectuadas por excavadores clandestinos a partir de los cortes antiguos. El cribado de este sedimento y la posterior regularización de los cortes proporcionó algunos restos óseos poco diagnósticos -un équido, cápridos y ciervo-y gran cantidad de fragmentos de carbón vegetal, pero ningún elemento industrial de origen humano. Su datación por radiocarbono ha mostrado que se trata de una ocupación medieval, situada entre los siglos XI-XIII en fechas

T. P., 57, n. ${ }^{\circ} 1,2000$ 
calibradas. Por debajo y por encima del nivel carbonoso se extienden sendos depósitos estalagmíticos blandos en la mayor parte de la zona excavada o regularizada, que alternan con lechos de arcilla de decalcificación. A continuación, y por toda la zona, se extendía un potente depósito de travertino poroso y blando. Se practicó un sondeo de $2 \mathrm{~m}^{2}$ a partir del fondo de la zanja, en el cual, tras otros $20 \mathrm{~cm}$ aproximadamente del mismo depósito, aparecían unas arcillas muy plásticas, en cuya base cambiaba la textura, haciéndose más arenosa y suelta. En el contacto de estos niveles se detectó una superficie con algunos cantos angulosos de caliza y, sobre todo, restos de industria lítica en sílex, incluyendo algunas lascas grandes y dos núcleos, uno de lascas laminares y otro de hojitas, junto con un raspador. Estos materiales estaban acompañados de restos de fauna, incluyendo herbívoros de gran talla (posiblemente bisonte o gran bóvido) y cérvidos. En este punto se detuvo la excavación de la zona, pero un testigo extraído mediante una sonda sacamuestras para análisis sedimentológico revela que al menos los depósitos arcillosos continúan con más de un metro de potencia.

En Abril de 1997, tras la primera campaña de excavación, se llevó a cabo un programa de prospección geofísica a cargo de Jaume Clapés y Raúl Osorio (Universitat Politécnica de Barcelona), destinada a evaluar la potencia del depósito sedimentario -que ya se revelaba como excepcional-y la posible presencia de zonas de desprendimiento de bloques, la extensión en superficie de los hogares ligados a las ocupaciones superficiales del Neolítico y Calcolítico de la zona de entrada, y la extensión y delineación de las zonas revueltas que se apreciaron durante la excavación de la zona interior del vestíbulo. El objetivo era el de poder tomar decisiones sobre la estrategia general a seguir en la excavación en años sucesivos.

Para ello se utilizaron tres técnicas distintas: resistividad eléctrica, magnetometría y georradar (GPR), cada una con la función de detectar un tipo concreto de anomalías previsibles según las hipótesis iniciales. Los resultados señalaron la presencia de un potente depósito sedimentario en el orden de los 8-10 $\mathrm{m}$ de espesor posible en la zona de entrada y vestíbulo, una menor alteración de lo previsto en este último, y una prolongación de los hogares de los niveles superiores del sondeo, de cronología calcolítica, hacia el centro de la galería, en la zona entonces no excavada, si bien su densidad se reducía a los lados inmediatos de la zona de trabajo de 1996. A esto se añadía la aparente ausencia de caos de bloques en esa potente serie sedimentaria.

Las características del depósito están siendo analizadas por W. Farrand (University of Michigan), en tanto que los aspectos relacionados con la micromorfología de los niveles del yacimiento y la edafología del entorno del mismo corre a cargo de M.A. Courty y N. Fedoroff (Institut National Agronomique de Paris). Geomorfólogos de la Universidad de Cantabria (J.C. García Codrón y E. Serrano) están analizando los aspectos ligados a la evolución cuaternaria de los valles fluviales inmediatos y las huellas de glaciarismo de sus cabeceras, así como la evolución del propio karst del Monte Pando, para comprender mejor los procesos de formación de la cueva y secuencia sedimentaria del Mirón, que incluye una terraza fósil muy antigua conservada en la galería interior. B. Ellwood (Lousiana State University) ha tomado varias series de muestras de sedimento para establecimiento de variaciones de susceptibilidad magnética que se correlacionan con cambios climáticos a lo largo de toda la secuencia.

\section{CRONOLOGÍA Y ATRIBUCIÓN DE LOS NIVELES}

La secuencia de ocupación de la Cueva del Mirón es una de las más completas excavadas hasta ahora en la Región Cantábrica, abarcando hasta este momento el intervalo 19000-3500 BP sin hiatos aparentes de sedimentación ni largos períodos de desocupación si exceptuamos el período 8000-6000 BP (Fig. 3). La lista completa de dataciones se recoge en la tabla 1 y las dataciones calibradas de los niveles de Prehistoria reciente, calibradas utilizando la curva decenal (Stuiver et alii, 1998) calculadas mediante el programa de calibración CALIB, rev. 4.1.2, en la tabla 2. La mayor parte de las dataciones, como se aprecia en la tabla 1 , se han obtenido mediante el procedimiento convencional de datación, y han sido realizadas por los Dres. H. Kruger y A. Cherkinsky (Geochron Laboratories, Cambridge, USA), si bien algunas muestras requirieron un conteo extendido por la escasa cantidad de material datable, en tanto que las fechas AMS se han realizado en el Lawrence Livermore Laboratory, en California, por tratarse de muestras demasiado pequeñas para aplicar el método convencional.

En este momento contamos con un total de 40 fechas para los niveles de la Cueva del Mirón, lo que la convierte en la secuencia continua mejor datada 


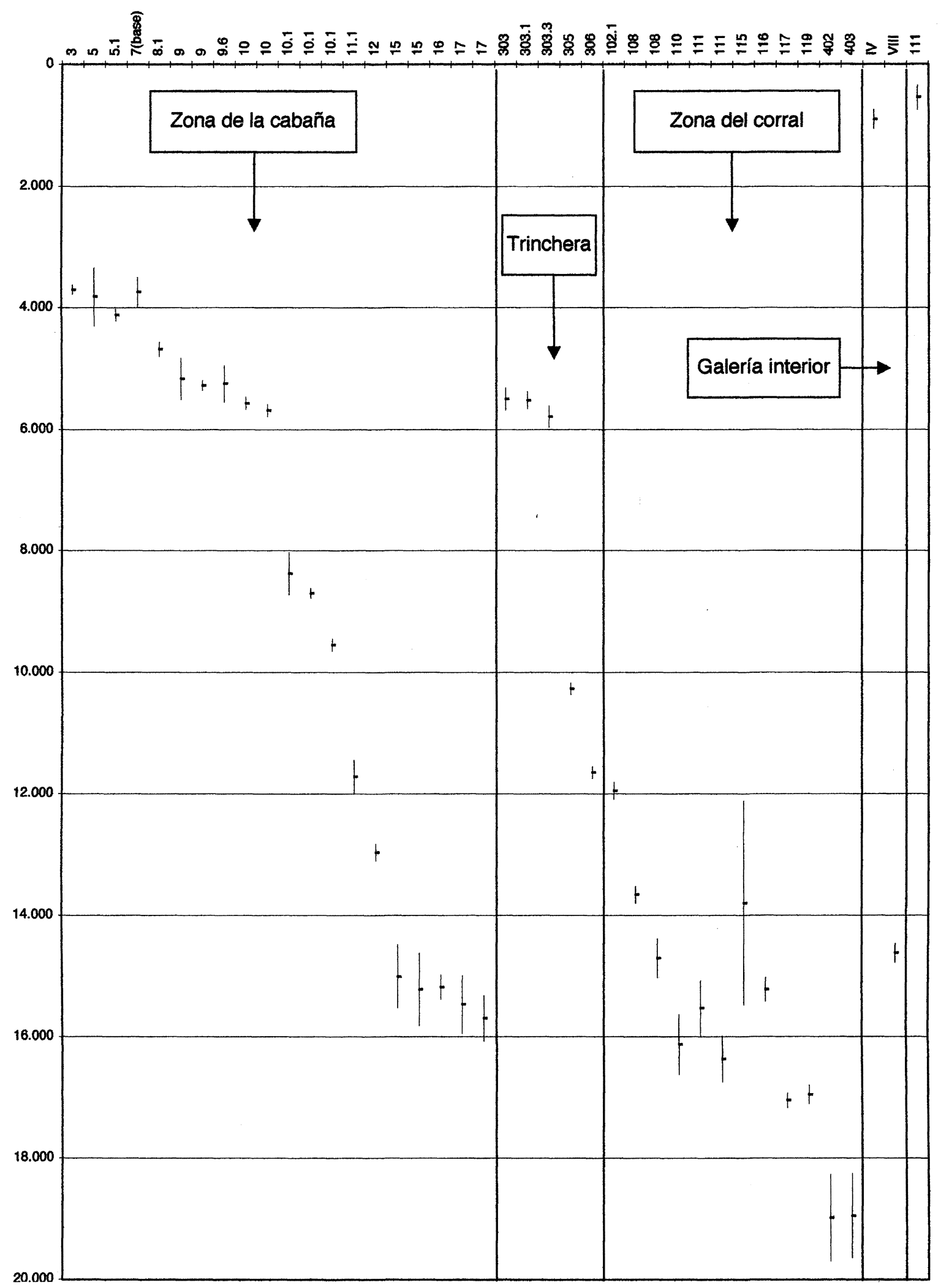

Fig. 3. Cueva del Mirón. Dataciones absolutas del conjunto del yacimiento.

T. P., 57, n. ${ }^{\circ} 1,2000$ 


\begin{tabular}{|c|c|c|c|c|c|c|c|c|c|c|}
\hline Nivel & Cuadro & Tramo & Atribución & Fecha BP & sd & Referencia & Mat. & Proc. & $d^{13} C$ & Año \\
\hline \multicolumn{11}{|c|}{ Zona de la cabaña: } \\
\hline 3 & $\mathrm{H} 2 \mathrm{a}$ & 4 & Calcolítico/Bronce & 3.700 & 40 & (GX-25851) & $\mathrm{C}$ & AMS & -26.2 & 1999 \\
\hline 5 & $\mathrm{~J} 2 \mathrm{a}$ & 5 & Calcolítico & 3.820 & 240 & $(\mathrm{GX}-22127)$ & $\mathrm{C}$ & conv & -28.1 & 1996 \\
\hline 5.1 & $\mathrm{I} 3 \mathrm{~d}$ & 4 & Calcolítico & 4.120 & 50 & $(\mathrm{GX}-22130)$ & $\mathrm{C}$ & AMS & -26.6 & 1996 \\
\hline 7(base) & $\mathrm{H} 2 \mathrm{~d}$ & 14 & Calcolítico & 3.740 & 120 & $(G X-24460)$ & $\mathrm{C}$ & conv & -26.4 & 1998 \\
\hline 8.1 & $\mathrm{I} 3 \mathrm{~b}$ & 13 & Neolítico & 4.680 & 60 & $(\mathrm{GX}-22131)$ & $\mathrm{C}$ & AMS & -26.1 & 1996 \\
\hline 9 & $\mathrm{~J} 2 \mathrm{a}$ & 8 & Neolítico & 5.170 & 170 & $(\mathrm{GX}-22128)$ & $\mathrm{C}$ & conv & -24.8 & 1996 \\
\hline 9 & $\mathrm{H} 4 \mathrm{a}$ & 22 & Neolítico & 5.280 & 40 & $(\mathrm{GX}-24461)$ & $\mathrm{C}$ & AMS & -26.0 & 1998 \\
\hline 9.6 & I4a & 22 & Neolítico & 5.250 & 150 & (GX-24462) & $\mathrm{C}$ & conv & -25.1 & 1998 \\
\hline 10 & $\mathrm{I} 3 \mathrm{a}$ & 19 & Neolítico & 5.570 & 50 & $(\mathrm{GX}-23414)$ & $\mathrm{C}$ & AMS & -25.7 & 1997 \\
\hline 10 & $\mathrm{I} 3 \mathrm{c}$ & 19 & Neolítico & 5.690 & 50 & $(\mathrm{GX}-23413)$ & $\mathrm{C}$ & AMS & -25.5 & 1997 \\
\hline 10.1 & $\mathrm{~J} 4 \mathrm{a}$ & 34 & ¿Mesolítico? & 8.380 & 175 & $(G X-24463)$ & $\mathrm{C}$ & conv & -24.7 & 1998 \\
\hline 10.1 & $\mathrm{I} 4 \mathrm{~b}$ & 28 & ¿Mesolítico? & 8.700 & 40 & $(\mathrm{GX}-25852)$ & $\mathrm{C}$ & AMS & -23.7 & 1999 \\
\hline 10.1 & $\mathrm{I} 4 \mathrm{c}$ & 29 & ¿Mesolítico? & 9.550 & 50 & $(\mathrm{GX}-24464)$ & $\mathrm{C}$ & AMS & -24.1 & 1998 \\
\hline 11.1 & I3 & 25 & Aziliense & 11.720 & 140 & $(\mathrm{GX}-23391)$ & $\mathrm{H}$ & conv & -21.8 & 1997 \\
\hline 12 & $\mathrm{~J} 2 \mathrm{c}$ & 12 & Magdaleniense Superior & 12.970 & 70 & $(\mathrm{GX}-22128)$ & $\mathrm{C}$ & AMS & -24.8 & 1996 \\
\hline 15 & $\mathrm{I} 3 \mathrm{a}$ & 43 & ¿Magdaleniense Inferior? & 15.010 & 260 & $(\mathrm{GX}-23392)$ & $\mathrm{H}$ & conv & -22.1 & 1997 \\
\hline 15 & I3d & 43 & ¿Magdaleniense Inferior? & 15.220 & 300 & $(\mathrm{GX}-23393)$ & $\mathrm{H}$ & conv & -25.6 & 1997 \\
\hline 16 & $\mathrm{I} 3 \mathrm{a}$ & 44 & Magdaleniense Inferior & 15.180 & 100 & $(\mathrm{GX}-23415)$ & $\mathrm{H}$ & AMS & -19.7 & 1997 \\
\hline 17 & $\mathrm{~J} 3 \mathrm{~d}$ & 32 & Magdaleniense Inferior & 15.470 & 240 & $(\mathrm{GX}-24466)$ & $\mathrm{H}$ & conv & -19.7 & 1998 \\
\hline 17 & $\mathrm{~J} 2 \mathrm{c}$ & 20 & Magdaleniense Inferior & 15.700 & 190 & $(\mathrm{GX}-25856)$ & $\mathrm{C}$ & conv & -26.1 & 1999 \\
\hline \multicolumn{11}{|c|}{ Trinchera del centro del vestíbulo: } \\
\hline 303 & L5a-d & 13 & Neolítico & 5.500 & 90 & $(\mathrm{GX}-25854)$ & $\mathrm{C}$ & conv & -25.8 & 1999 \\
\hline 303.1 & L5a & 14 & Neolítico & 5.520 & 70 & $(\mathrm{GX}-25855)$ & $\mathrm{C}$ & conv & -26.2 & 1999 \\
\hline 303.3 & L5a-d & 16 & Neolítico & 5.790 & 90 & $(\mathrm{GX}-25856)$ & $\mathrm{C}$ & conv & -26.1 & 1999 \\
\hline 305 & $\mathrm{P} 6 \mathrm{a}$ & 9 & ¿Aziliense? & 10.270 & 50 & $(\mathrm{GX}-24467)$ & $\mathrm{H}$ & AMS & -20.7 & 1998 \\
\hline 306 & $\mathrm{P} 6 \mathrm{a}$ & 11 & ¿Aziliense? & 11.650 & 50 & $(\dot{G} X-24468)$ & $\mathrm{H}$ & AMS & -20.7 & 1998 \\
\hline \multicolumn{11}{|c|}{ Zona del corral: } \\
\hline 102.1 & T8d & 3 & ¿Magdaleniense Final? & 11.950 & 70 & $(G X-23417)$ & $\mathrm{H}$ & AMS & -25.8 & 1997 \\
\hline 108 & $\mathrm{~V} 8 \mathrm{c}$ & 4 & ¿Magdaleniense? & 13.660 & 70 & $(\mathrm{GX}-22703)$ & $\mathrm{H}$ & AMS & -21.8 & 1996 \\
\hline 108 & V8d & 5 & ¿Magdaleniense Medio? & 14.710 & 160 & $(\mathrm{GX}-23397)$ & $\mathrm{H}$ & conv & -21.7 & 1997 \\
\hline 110 & V8d & 8 & Magdaleniense Inferior & 16.130 & 250 & $(\mathrm{GX}-23396)$ & $\mathrm{H}$ & conv & -21.6 & 1997 \\
\hline 111 & $\mathrm{U} 7 \mathrm{a}$ & 20 & Magdaleniense Inferior & 15.530 & 230 & (GX-24468) & $\mathrm{H}$ & conv & -20.6 & 1998 \\
\hline 111 & V8d & 14 & Magdaleniense Inferior & 16.370 & 190 & $(\mathrm{GX}-23395)$ & $\mathrm{H}$ & conv & -20.9 & 1997 \\
\hline 115 & V8d & 19 & Magdaleniense Inferior & 13.800 & 840 & (GX-23394) & $\mathrm{H}$ & conv & -24.6 & 1997 \\
\hline 116 & V8d & 20 & Magdaleniense Inferior & 15.220 & 100 & $(\mathrm{GX}-23416)$ & $\mathrm{H}$ & AMS & -20.8 & 1997 \\
\hline 117 & V8a-d & 24 & Magdaleniense Inferior & 17.050 & 60 & $(\mathrm{GX}-25857)$ & $\mathrm{C}$ & AMS & -24.5 & 1999 \\
\hline 119 & V8a-c & 28 & ¿Magdaleniense inferior? & 16.960 & 80 & $(\mathrm{GX}-25858)$ & $\mathrm{C}$ & AMS & -23.4 & 1999 \\
\hline $125(=402)$ & W10d & 4 & Solutrense & 18.980 & 360 & $(\mathrm{GX}-24470)$ & $\mathrm{H}$ & conv & -20.4 & 1998 \\
\hline $126(=403)$ & W10d & 5 & Solutrense & 18.950 & 350 & $(\mathrm{GX}-24471)$ & $\mathrm{H}$ & conv & -20.2 & 1998 \\
\hline \multicolumn{11}{|c|}{ Galería interior de la cueva: } \\
\hline IV & 8Q-9Q & & Medieval & 900 & 80 & $(\mathrm{GX}-22129)$ & $\mathrm{C}$ & conv & -25.7 & 1996 \\
\hline VIII & $12 \mathrm{R}$ & & ¿Magdaleniense Medio? & 14.620 & 80 & $(\mathrm{GX}-22347)$ & $\mathrm{C}$ & AMS & -25.7 & 1996 \\
\hline \multicolumn{11}{|l|}{ Anómalas: } \\
\hline 111 & $\mathrm{U} 7 \mathrm{~b}$ & 20 & & 540 & 100 & $(\mathrm{GX}-24465)$ & $\mathrm{C}$ & conv & -26.4 & 1998 \\
\hline
\end{tabular}

Tab. 1. Dataciones absolutas de la Cueva del Mirón. Clave: Mat. = material datado $(\mathrm{C}=$ carbón, $\mathrm{H}=$ hueso $)$; Proc. $=$ procedimiento de datación (conv = datación de radiocarbono convencional). 


\begin{tabular}{|l|r|r|r|r|r|r|}
\hline Referencia & Nivel & Fecha BP & \pm & \multicolumn{3}{|c|}{ Años Cal BC } \\
\hline & & & & $+2 s$ & $-2 s$ & Edades calibradas \\
\hline GX-25851 & 3 & 3.700 & 40 & 2902 & 1957 & $2129,2082,2043$ \\
\hline GX-22127 & 5 & 3.820 & 240 & 2902 & 1622 & $2283,2249,2232,2218,2211$ \\
\hline GX-22130 & 5.1 & 4.120 & 50 & 2879 & 2496 & $2834,2819,2662,2648,2625$ \\
\hline GX-24460 & 7 & 3.740 & 120 & 2472 & 1777 & 2141 \\
\hline GX-22131 & 8.1 & 4.680 & 60 & 3637 & 3351 & $3501,3431,3379$ \\
\hline GX-24461 & 9 & 5.170 & 170 & 4345 & 3641 & 3973 \\
\hline GX-24462 & 9.6 & 5.280 & 40 & 4225 & 3981 & $4216,4203,4136,4134,4045$ \\
\hline GX-23414 & 10 & 5.570 & 50 & 4497 & 4334 & $4041,4016,4003$ \\
\hline GX-23413 & 10 & 5.690 & 50 & 4686 & 4372 & $4439,4423,4363$ \\
\hline GX-25854 & 303 & 5.500 & 90 & 4516 & 4052 & $4519,4511,4501$ \\
\hline GX-25855 & 303.1 & 5.520 & 70 & 4493 & 4245 & 4342 \\
\hline GX-25856 & 303.3 & 5.790 & 90 & 4846 & 4409 & 4349 \\
\hline GX-24463 & 10.1 & 8.380 & 175 & 7750 & 7051 & $7685,4676,4675,4635,4621$ \\
\hline GX-25852 & 10.1 & 8.700 & 40 & 7939 & 7598 & $7728,7718,7713,7697,7680$ \\
\hline GX-24464 & 10.1 & 9.550 & 50 & 9208 & 8693 & $9107,9097,9095,9070,9051,9012,8810$ \\
\hline
\end{tabular}

Tab. 2. Fechas calibradas de los niveles postpaleolíticos de la Cueva del Mirón (curva INTCAL98, Stuiver et alii, 1998).

del Cantábrico. Cabe señalar que tan sólo una de las fechas obtenidas ha sido considerada como claramente anómala, sin duda producida por un carbón moderno caído del corte y que parece apuntar a la continuidad de uso del vestíbulo dela cueva en época histórica. La serie de ocupaciones de la Prehistoria reciente de la zona de la cabaña cuenta con diez dataciones, que fechan respectivamente el entorno inmediato del hallazgo de un punzón biapuntado de cobre y diversas cerámicas (Nivel 3: 3700 $\pm 40 \mathrm{BP}$ ), hogares de cubeta calcolíticos con puntas de aletas y pedúnculo (Nivel 5: $3820 \pm 240 \mathrm{BP}$ ) y de base convexa (Nivel 5.1:4120 $\pm 50 \mathrm{BP}$ ), o el estrato en el cual se excavan la mayoría de esos hogares (Nivel 7: $3740 \pm 120 \mathrm{BP})$. La proximidad y casi coincidencia estadística de estas fechas, junto con los datos de la micromorfología, parecen mostrar que esta parte del depósito se acumuló con gran rapidez por una ocupación intensiva de la cueva como espacio doméstico y de estabulación. La secuencia neolítica se abre con el nivel 8 y el lentejón $8.1(4680 \pm 60$ BP $)$ y continúa con los niveles $9(5170 \pm 170$ y $5280 \pm 40 \mathrm{BP})$, $9.6(5250 \pm 150 \mathrm{BP})$ y la parte superior del paquete $10(5570 \pm 50$ y $5690 \pm 50 \mathrm{BP})$, donde forman pequeños hogares. La continuación de estas ocupaciones hacia el interior del vestíbulo se pone de manifiesto en la estratigrafía del sondeo, con tres fechas de los niveles o lentejones 303 (5500 $\pm 90 \mathrm{BP}), 303.1$ $(5520 \pm 70 \mathrm{BP})$ y $303.3(5790 \pm 90 \mathrm{BP})$. Estos estra- tos bajo el nivel 8 incluyen, junto con hogares superficiales y numerosos restos de fauna, contados microlitos geométricos (trapecios y segmentos de círculos, estos últimos con retoque abrupto o en doble bisel), y vasos cerámicos de paredes finas, pastas cuidadas y superficies alisadas, siempre sin decoración.

La continuación en profundidad del nivel 10, el paquete 10.1 , individualizado a partir de la campaña de 1997, se formó (como el 10) bajo condiciones de elevada humedad traducida en muestras de encharcamiento y goteo, un período en el que la cueva recibe visitas esporádicas que dejan como testimonio algunos materiales líticos, contados restos de fauna y escasos carbones que han proporcionado tres fechas para momentos sucesivos de $8380 \pm 175$, $8700 \pm 40$ y $9550 \pm 50$ BP. El hiato de fechas de ocupaciones para el intervalo 5800-8300 BP es especialmente significativo porque corresponde con momentos en que se cuenta con varias fechas para la zona de la cuenca baja delAsón o la costa inmediata, en las cuevas de La Fragua, La Trecha (González Morales, 1995, 1999b) y La Garma (V.V.A.A., 1999: 106), o la ocupación al aire libre del Ilso de Hayas (Serna, 1997), y su final coincide con la supuesta ocupación mesolítica muy tardía de la inmediata cueva de Tarrerón, frente a la del Mirón, con elementos que delataban el contacto con la costa (Apellániz, 1971). Esperamos disponer de los datos 
completos de estas ocupaciones y de las características de formación de este depósito para poder verificar las hipótesis alternativas que se plantean para explicar el aparente abandono de las áreas interiores de la zona en parte del Mesolítico (González Morales, 1999a).

La datación de los estratos 305 y 306 del sondeo central ha permitido verificar las observaciones estratigráficas, que los situaban entre el techo de la serie de niveles intactos del corral y la base del nivel 10.1 de la cabaña, posición ahora plenamente confirmada y que resulta esencial para correlacionar los estratos de las distintas zonas de la cueva en el sentido recogido más arriba. Otro factor importante a tener en cuenta es que a partir del nivel 304 los estratos se extienden por la totalidad del vestíbulo, permitiendo el análisis de las formas diferenciales de ocupación del espacio y organización interna en las distintas fases de uso de la cueva a lo largo del Paleolítico Superior. Las ocupaciones del Magdaleniense superior y medio -en términos de unidades cronoestratigráficas convencionales- son relativamente pobres en materiales líticos y óseos en la entrada de la cueva, pero los niveles 104 a 107 -y sus diversos lentejones-en la zona del corral han proporcionado más de 27000 restos de talla y 343 útiles retocados; sin embargo, la fuerte remoción por madrigueras que presenta esa zona de la estratigrafía obliga a tomar precauciones especiales en cuanto a muestreo para datación, por lo cual estamos aún pendientes de una mayor precisión sobre su fecha. El nivel 108 es especialmente rico (en este momento casi 30.000 restos de talla y 540 útiles retocados), y contamos con dos fechas de $13660 \pm 70$ y $14710 \pm 160 \mathrm{BP}$, problemáticas en cierta medida porque aparecen asociadas a instrumentos de asta -como las azagayas de aplastamiento central desplazado a la base "tipo Castillo" o de sección cuadrada "tipo Juyo"- que se han venido atribuyendo a etapas arcaicas del Magdaleniense cantábrico. Aparte de la fecha de $13800 \pm 840$ del nivel 115 , con una elevada desviación que la hace escasamente utilizable, las variaciones del resto de las fechas para el conjunto de estratos 110-116, muy similares entre sí en cuanto a textura y contenido, parecen indicar una acumulación rápida, y todo el paquete de tierras orgánicas oscuras 108-119 refleja una acción antrópica intensa, que incluye posibles estructuras constructivas, cubetas y hogares, y no son de descartar otras remociones. Hay que tener en cuenta que esos niveles -de 112 en adelante- solamente se han excavado en el sondeo del cuadro V8, de algo menos de un metro cuadrado. A $2 \mathrm{~m}$ de distancia, en el sondeo al pie de la pendiente que conduce al interior de la cueva, los niveles solutrenses han proporcionado fechas perfectamente compatibles con tal atribución, llevando la cronología de las ocupaciones de la Cueva del Mirón al límite provisional de 19000 años BP. En la zona de entrada contamos con una serie coherente de cinco fechas que sitúa la formación de los niveles 15 a 17 , con suelos de ocupación definidos y materiales asig-nados al Magdaleniense inferior, entre 15000 y $15700 \mathrm{BP}$.

Por último, las fechas obtenidas en el sondeo de la galería interior de la cueva muestran que esa zona, al límite entre la penumbra y la oscuridad, fue visitada al menos durante la Baja Edad Media, cuando sirvió probablemente de refugio, y en algún momento del Magdaleniense (14620 \pm 80 BP).

\section{LOS MATERIALES ARQUEOLÓGICOS Y SU ESTUDIO}

A lo largo de estas campañas se ha venido realizando la clasificación preliminar de los restos de talla líticos y de los útiles retocados, si bien está pendiente de completarse su revisión definitiva. Hasta el presente se han contabilizado 155077 restos de talla y 2342 útiles; de los primeros se presenta un avance en la tabla 3, con la idea de ilustrar su distribución por niveles. Todos los restos mayores de $1 \mathrm{~cm}$ de dimensión máxima han sido clasificados teniendo en cuenta su materia prima (sobre un muestrario de unas 40 variedades diferentes) y pesados individual o colectivamente para estimar el uso de distintos materiales, y todos los útiles y las piezas recogidas individualmente y coordenadas en el yacimiento se han medido, lo cual nos está proporcionando una importante base de datos para desarrollar estudios sobre los procesos de producción del instrumental lítico. J.E. González Urquijo (Universidad de Cantabria) y J.J. Ibáñez están trabajando en el estudio de huellas de uso con vistas a identificar los procesos de trabajo implicados. La industria ósea y la cerámica están actualmente en proceso de restauración, reconstitución (por Z. San Pedro) y estudio. M. ${ }^{a}$ L. Ramos (Universidad de Cantabria) prepara una aproximación experimental a los procesos de manufactura de la cerámica, a partir de los análisis de pastas y cocción desarrollados por F. Guitián (Instituto de Cerámica, Universidad de Santiago de Compostela). 


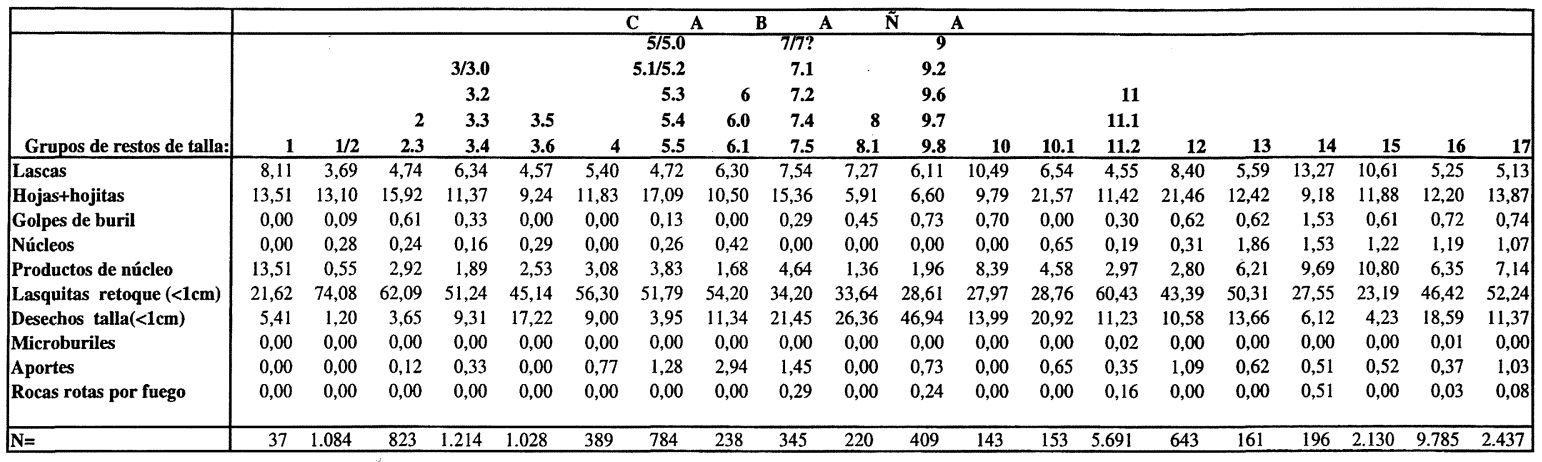

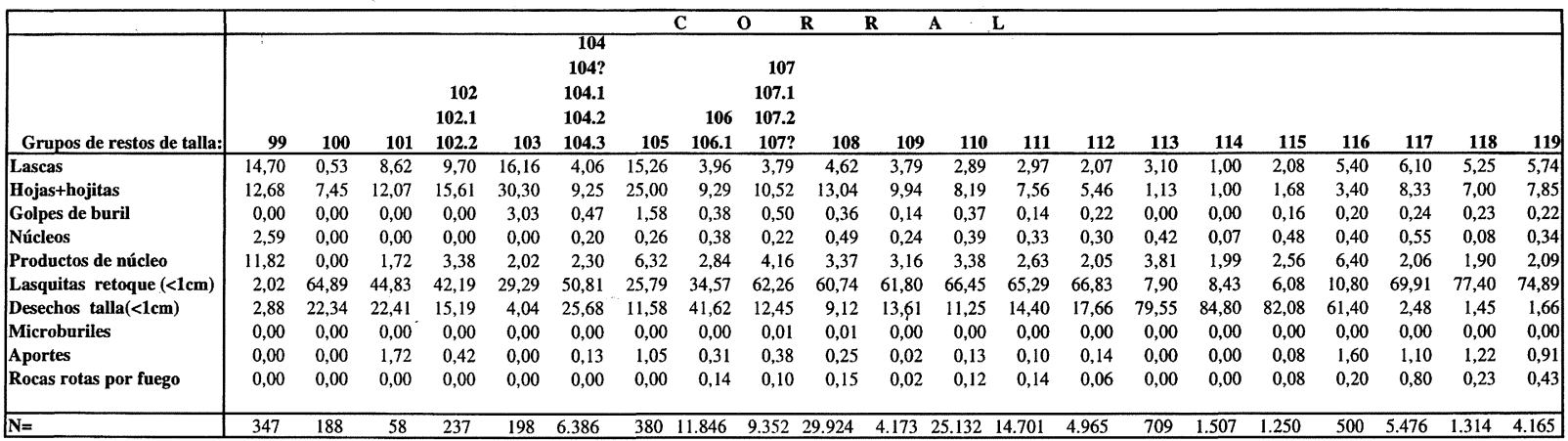

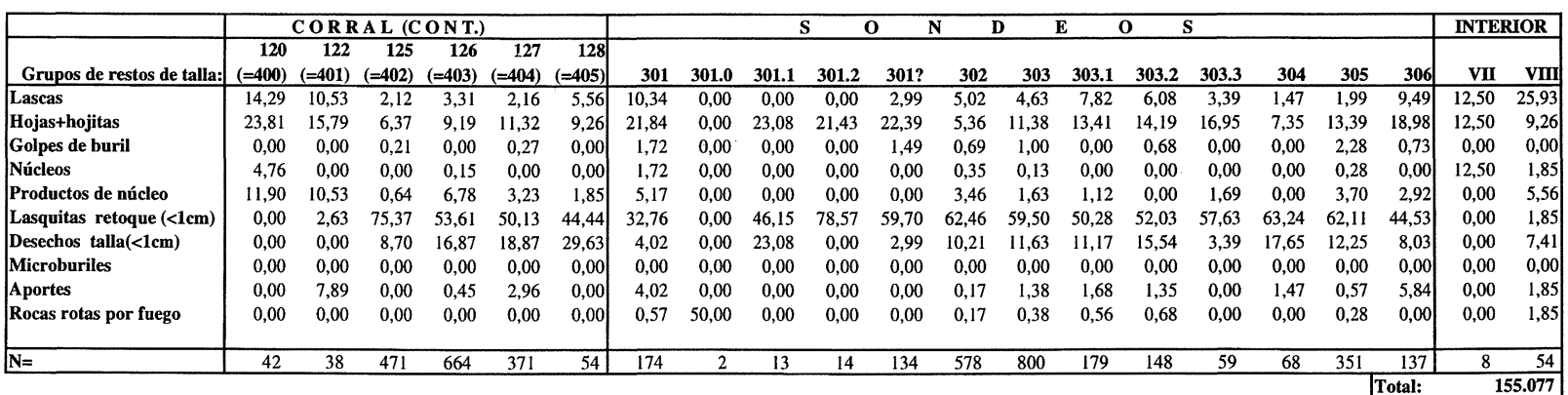

Tab. 3. Cueva del Mirón: porcentajes de tipos de restos de talla por niveles agrupados. Campañas 1996-1999.

Los restos de fauna de macromamíferos están siendo estudiados por J. Altuna y Koro Mariezkurrena (Sociedad de Ciencias Aranzadi), en tanto que la microfauna corre a cargo de G. Cuenca (Universidad de Zaragoza) y su equipo. La aparición de abundantes restos de salmónidos ha servido de base para el desarrollo de un programa de investigación para la caracterización genética de los mismos por el equipo de biólogos del Centro Ictiológico de Arredondo (Cantabria), integrado por C. García de Leániz, S. Consuegra y A. Serdio. M.J. Iriarte (Universidad de Santiago de Compostela y Sociedad de Ciencias Aranzadi) realiza actualmente el estudio de la secuencia polínica del Mirón, en tanto que L. Zapata (Cambridge University) y L. Peña están analizando las muestras de macrorrestos vegetales procedentes de la flotación.

\section{CONCLUSIÓN}

Los resultados obtenidos hasta el presente en la Cueva del Mirón y las investigaciones derivadas de los mismos están suministrando información de calidad excepcional para contrastar distintas hipótesis sobre las sociedades prehistóricas de la zona, así como otros varios aspectos complementarios, y las perspectivas que ofrece su depósito parecen lejos de agotarse. A la vez, nuevas cuestiones se plantean a partir de estos hallazgos que obligan a reconsiderar aspectos del diseño inicial de la investigación. La intención de los autores es completar en breve la primera monografía, correspondiente a los niveles de la Prehistoria reciente, para su publicación y dar así a conocer con mayor detalle-y someter a debate-los fundamentos de nuestras conclusiones. 


\section{AGRADECIMIENTOS}

Las investigaciones en la Cueva del Mirón han venido siendo financiadas por la Fundación Marcelino Botín (1996-99), la L.S.B. Leakey Foundation (1996-97), la National Geographic Society (199798), la National Science Foundation (1999) y la University of New Mexico (1996-99), y ha contado con el apoyo directo y continuo del Ayuntamiento de Ramales de laVictoria, que pone a disposición de nuestro equipo locales e instalaciones para laboratorio de campo y del Colegio Público "Príncipe de Asturias" de Ramales, que facilita las instalaciones de su Aula de Informática para uso igualmente como laboratorio. La investigación complementaria sobre la geomorfología local y las áreas de captación de materias primas está financiada a través de un proyecto trianual de la Dirección General de Enseñanza Superior del Ministerio de Educación y Cultura (Programa Sectorial de Promoción General del Conocimiento, Proyecto PB96-0442), y la Consejería de Ganadería, Pesca y Agricultura del Gobierno de Cantabria está financiando una línea de trabajo en genética a partir de los restos fósiles de salmónidos, como antes señalamos. Joaquín Eguizábal, responsable de la Cueva de Covalanas, ha sido un colaborador esencial tanto para la adecuada conservación de la cueva como para el buen funcionamiento del equipo en Ramales.A ellos, y a todos cuantos han venido colaborando en las distintas campañas de excavación y estudio (alumnos de la Universidad de Cantabria y New Mexico, así como de otras muchas universidades españolas, europeas o americanas), debemos nuestro agradecimiento.

\section{BIBLIOGRAFÍA}

Alcalde del Río, H. (1906): Las Pinturas y Grabados de las Cavernas Prehistóricas de la Provincia de Santander. Blanchard y Arce. Santander.

Alcalde del Río, H.; Breuil, H. y Sierra, L. (1911): Les cavernes de la Région Cantabrique. Imp. V. Chêne. Monaco.

ApellánIz, J.M. (1971): "El Mesolítico de la Cueva de Tarrerón y su datación por el C-14”. Munibe, 23: 91-104.

CABré, J. (1915): ElArte Rupestre en España. Comisión de Investigaciones Paleontológicas y Prehistóricas. Madrid.

GonzÁlez Morales, M.R. (1995): "La transición al Holoceno en la Región Cantábrica: el contraste con el modelo del mediterráneo español.” En V. Villaverde Bonilla (ed.): Los últimos cazadores. Transformaciones culturales y económicas durante el Tardiglaciar y el inicio del Holoceno en el ámbito mediterráneo. Instituto de Cultura "Juan Gil Albert". Alicante: 63-78.

- (1999a): "Costa e Interior: Algunas Observaciones sobre el Uso de las Cuevas en el Mesolítico". De Oriente a Occidente. Homenaje al Dr. Emilio Olávarri. Universidad Pontificia de Salamanca. Salamanca: 237-246.

- (1999b): "La Prehistoria en Santoña". Monte Buciero, 2: 17-28.

González Morales, M.R. y Straus, L.G. (1997): “La Prehistoria del Valle del Asón: Excavaciones en la Cueva del Mirón. La campaña de 1996.” En R. de Balbín Behrmann y P. Bueno (eds.): II Congreso de Arqueología Peninsular. Paleolítico y Epipaleolítico, I. Fundación Rei Afonso Henriques. Zamora: 119-131.

Obermaier, H. (1916): El Hombre Fósil. Comisión de Investigaciones Paleontológicas y Prehistóricas. Madrid.

- (1924): Fossil Man in Spain. Yale University Press. New Haven.

SERnA, M. ${ }^{a}$ R. (1997): "Neolitización y megalitismo en la cornisa cantábrica: El yacimiento de Guriezo-Hayas". En R. de Balbín Behrmann y P. Bueno (eds.): II Congreso de Arqueología Peninsular. Neolítico, Calcolítico y Bronce, II. Fundación ReiAfonso Henriques. Zamora: 199-206.

SiERRA, L. (1908): "Notas para el mapa paletnográfico de la Provincia de Santander". Actas del Primer Congreso de Naturalistas Españoles. Zaragoza: 103-117.

Straus, L.G. y González Morales, M.R. (1996): "Preliminary excavations in El Mirón Cave (Ramales de laVictoria, Cantabria, Spain)". Old World Archceology Newsletter, 20: 14-18.

- (1997): "The RíoAsón Prehistoric Project: 1997 Excavations in El Mirón Cave (Ramales de laVictoria, Cantabria, Spain)". OldWorldArchaology Newsletter, 21: $1-11$.

- (1998a): "Report on the initial excavations in El Miron Cave (Ramales de la Victoria, Cantabria, Spain) with emphasis on the Magdalenian occupations". Journal of Iberian Archaology, 0:173-188.

- (1998b): "Tardiglacial Settlement in the Cantabrian Cordillera, Northern Spain”. Journal of Human Evolution, 34: A20-A21.

- (1998-99): “1998 Excavation Campaign in EL Mirón Cave (Ramales, Cantabria, Spain)". Old WorldArchaeology Newsletter, 21: 1-9.

- (1999): "The Fourth Excavation Campaign in 'El Mirón' Cave (Cantabria, Spain), 1999.” Old WorldArchaeology Newsletter, 22: 1-8.

Stuiver, M.; ReImer, P.J.; BARD, E.; BeCK, J.W.; BurR, G.S.; Hughen, K.A.; Kromer, B.; McCormac, F.G.; V.D. Plicht, J. y SPURK, M. (1998): "INICAL 98 Radiocarbon Age Calibration, 24,000-0 cal BP”. Radiocarbon, 40: 1041-1083.

V.V.A.A. (1999): La Garma. Un descenso al pasado. Gobierno de Cantabria y Universidad de Cantabria. Santander. 